\title{
Model Assisted Design and Simulation of a Pharmaceutical Batch Process; Manufacturing of Clopidogrel Bisulfate
}

\author{
M. Trifkovic, S. Cardoso, M. Sheikhzadeh, S. Rohani, S. Barghi \\ The Department of Chemical \& Biochemical Engineering, The University of Western \\ Ontario, London, Ontario, Canada, N6A 5B9
}

\begin{abstract}
A novel approach was taken in the fourth year design course for Chemical Engineers. A complex pharmaceutical process for the manufacturing of Clopidogrel Bisulfate (CPG), an anti-platelet drug, was successfully designed and simulated using AspenBatch ${ }^{\mathrm{TM}}$ and SuperPro Designer (SPD) software packages. Two models were applied in the design of the crystallizer and dryer, which provided the optimal cooling rate for crystallization and the surface area of the dryer. The solubility of CPG in acetone was determined experimentally by Attenuated Total Reflectance Fourier Spectroscopy (ATR-FTIR) instrument. Multifunctional equipment were designed to reduce the production and maintenance costs.
\end{abstract}

\section{Introduction}

Clopidogrel (CPG) is a potent anti-platelet drug, which was launched in 1997 by SanofiSynthelab with the brand name Plavix ${ }^{\mathrm{TM}}$ [1]. It is indicated for the prevention of vascular thrombotic events in patients at risk. Clopidogrel Bisulfate is inactive in vitro, and a hepatic biotransformation is necessary to express the full anti-aggregating activity of the drug. Six different polymorphs have been identified so far, but only Form I and Form II are used in pharmaceutical formulations as they are the only polymorphs that exhibit bioavailability [2]. The chemical structure of CPG (methyl $(+)-(\mathrm{S})-\propto-(0-$ chlorophenyl)-6,7-dihydrothiento[3,2-c]pyridine$5(4 \mathrm{H})$-acetate), in $2 \mathrm{D}$ and $3 \mathrm{D}$, is shown below in Figure 1.

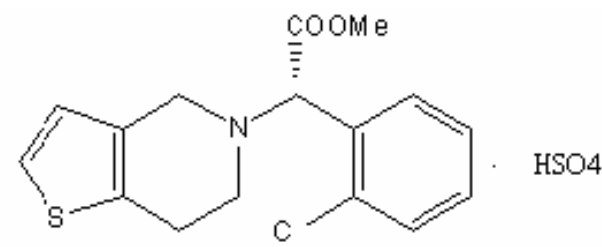

Figure 1. Chemical Structure of Clopidogrel Molecule

The economic burden worldwide in treating patients who have suffered and survived a stroke has been extensively studied and found to average $3 \%$ to $5 \%$ of all national healthcare costs [3]. It was also reported that patients treated with Plavix $^{\mathrm{TM}}$, in combination with other anti-platelet drugs, experience the greatest reduction in occurrence of stroke [4]. Compared with aspirin, clopidogrel reduced the combined risk of ischemic stroke, myocardial infarction or vascular death by $8.7 \%$ [5]. Gerschutz et al. [6] showed that Plavix ${ }^{\mathrm{TM}}$ combined with Aspirin ${ }^{\mathrm{TM}}$ is more effective at reducing various detrimental vascular events compared to Aspirin ${ }^{\mathrm{TM}}$ alone. Overall, there was a $20 \%$ relative risk reduction $(95 \%$ CI of $10 \%-28 \%)$ for the Plavix ${ }^{\mathrm{TM}}$ treated group [6].

In this study, the design for the manufacturing of Clopidogrel Bisulfate was performed. The mathematical modeling is used often in practice to optimize the manufacturing process. In pharmaceutical industry, the reactor and the dryer are two important operational units, and thus were chosen for the detailed design in this study. The mathematical modeling approach was taken for the design and optimization of the crystallization process. Crystal size distribution (CSD) plays an important role in the quality of this anti-platelet drug. Solubility of the product and the temperature profile are important factors that affect the CSD [7]. The solubility of the $\mathrm{CPG}$ in solvent used for the crystallization process was determined experimentally by using Attenuated Total Reflection Fourier Transform Infrared Spectroscopy (ATR-FTIR) instrument.

Computer Aided Process Designed and simulation tools have been successfully used in 
the design and development of bulk synthetic pharmaceutical processes [8]. In this study, the manufacturing process was simulated using two well-known software packages for batch processes; SuperPro Designer and AspenBatch TM.

\section{Active Pharmaceutical Ingredient (API) Production}

The proposed plant capacity for the manufacturing of CPG is 91.25 million tablets per year, although the plant is considered for production of other active pharmaceutical ingredients. There are six chemical reactions involved in producing Clopidogrel Bisulfate from the raw materials.

\section{Process Description and Simulation with Software}

The following section describes the process and simulation with software packages.

The SPD is an advanced simulation and data management tool for modeling of complex, recipe-based batch processes such as those found in pharmaceutical industries. The tool supports a wide spectrum of engineering workflows over the full lifecycle of a process. Although not covered in this report, the software is very useful for secondary processes in pharmaceutical industry; such are packaging and solids processes. AspenBatch ${ }^{\mathrm{TM}}$ is recipe driven software package, which allows the user to develop a model by creating a text recipe. The modeling engine creates a Process Flow Diagram (PFD) as the output. Both software packages are not dynamic, but rather simple algebraic models, whose solution does not require integration of differential equations. This shortens the computation time and enables the user to evaluate a larger number of scenarios in a shorter period.

This report focuses on simulation of the primary process, or the production of the active pharmaceutical ingredient (Clopidogrel Bisulfate). The simulation of the entire process for manufacturing Clopidogrel Bisulfate is shown in Figure 2.

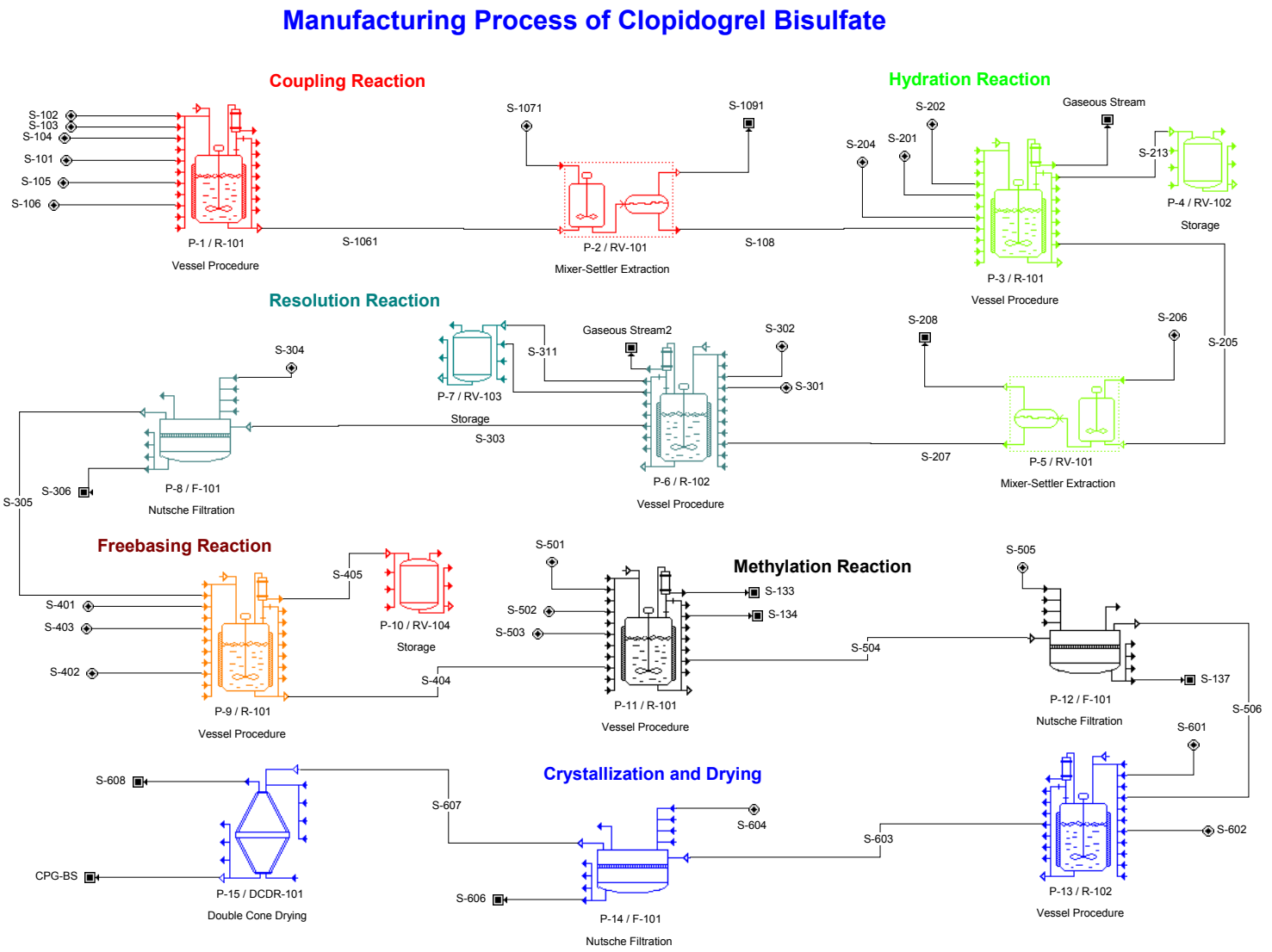

Figure 2. Process Flow Diagram (PFD) obtained from SuperPro Designer 


\subsection{Coupling Reaction (Strecker Synthesis)}

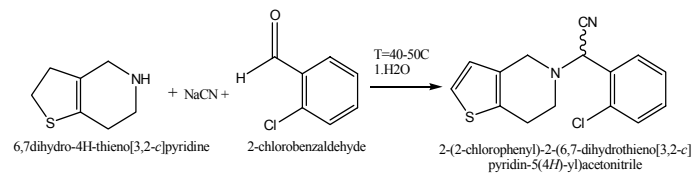

\section{Scheme 1. Reaction Mechanism for Strecker Synthesis (Step 1)}

The raw materials are received and put under quarantine until they pass testing criteria for purity. Sodium bisulfate (S-101) is mixed with purified water (S- 105) in the reactor (R-101). The reactor is not pressurized (although is still equipped with a pressure relief valve (PRV-101) as a safety precaution) and the addition of all reactants, and solvents is done under atmospheric pressure (approximately $101.3 \mathrm{KPa}$ ) and room temperature. Once the sodium bisulfate is dissolved, o-chlorobenzaldehyde (S-102) is pumped via into the same reactor, resulting in a white precipitate. 6,7 , dihydro-4-H thieno $[3,2$, c] pyridine (S- 103) is pumped into the reactor via and sodium cyanide (S-104) is also added to the reaction mixture. The reactor jacket temperature is maintained at the desired temperature range of $40-50{ }^{\circ} \mathrm{C}$ and maintained for 6 hours until the reaction is quenched with water (S-105) via pump. The product of this reaction is transferred by gravity into a receiving vessel (RV-101). Ethyl acetate (S-107) is pumped into the same receiving vessel and the mixture undergoes liquid-liquid extraction. The organic layer (S-108) is pumped back to the reactor while the aqueous layer is left in the receiving vessel (RV-101). Here the aqueous layer is extracted a second time to maximize the isolation of the product, which is then sent back to the reactor via a pump. The aqueous layer (S109 ) is sent to the waste treatment.

\section{Process Simulation of Step 1}

The process described above is simulated using both, SuperPro Designer (SPD) and AspenBatch ${ }^{\mathrm{TM}}$ software packages. The first step in the simulation is registering all the ingredients involved in the process. This can be done by retrieving the specific component from SPD pure component library, or by entering the physical data manually in user databank. The components were registered in a similar way in AspenBatch ${ }^{\mathrm{TM}}$.
The unit-operations in sequence needed to simulate the Step 1 are shown in Table 1.

Table 1. Unit operations for Step I

\begin{tabular}{|c|c|}
\hline $\begin{array}{c}\text { Equipment } \\
\text { Tag }\end{array}$ & Unit Operation \\
\hline \multirow{4}{*}{ R-101 } & Charge (Water) \\
\cline { 2 - 2 } & Charge (Sodium Bisulfate) \\
\cline { 2 - 2 } & Heat \\
\cline { 2 - 2 } & Agitate \\
\cline { 2 - 2 } & Charge (Chlorobenzaldehyde) \\
\cline { 2 - 2 } & Charge (6,7 dihydro-4H-pyridine) \\
\cline { 2 - 2 } & Batch Stoich. Reaction \\
\cline { 2 - 2 } & Charge (Water-Quenching) \\
\cline { 2 - 2 } & Transfer Out \\
\hline \multirow{4}{*}{ RV-101 } & $\begin{array}{c}\text { Mixer }- \text { Settler Extraction } \\
\text { (Extract - Intermediate I) }\end{array}$ \\
\hline
\end{tabular}

Each operation was initialized with the appropriate engineering data such are charge quantities, reaction stoichiometry, and scheduling relations for both software packages. The operation duration was fixed since it was known from the patent data [1]. Furthermore, the operation's start was specified either based on the beginning of the batch (charging the raw material), or related to the end of another operation (i.e. reaction was started at the end of charging of the reactants). Reaction operation required entering the reaction stoichiometry, and the extraction operation required a separation yield for each stream component. The reaction conversion was known from the patent data $(96 \%)$, and the separation was assumed to be perfect for the purpose of simplicity of the model

The quenching operation was not available in the SuperPro software, and thus water-quenching charge operation was used to compensate for this missing operation. The same operation was available in AspenBatch ${ }^{\mathrm{TM}}$.

\subsection{Efficient Hydration of Nitriles to Amides}

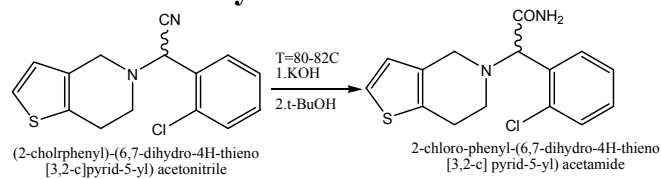

Scheme 2. Reaction Mechanism for Efficient Hydration of Nitriles to Amides (Step 2)

The excess solvent is first vaporized using vacuum conditions, and it is collected into the 
receiving vessel (RV-102). The product of the first step remains in the reactor where butyl alcohol (S-201) is pumped and $\mathrm{KOH}(\mathrm{S}-202)$ is added. This mixture is stirred vigorously, after which it is refluxed using the condenser for three hours at $80-82{ }^{\circ} \mathrm{C}$. The reactor jacket is then cooled (S-212) to $30{ }^{\circ} \mathrm{C}$ and purified water (S204) is pumped by to stop the reaction. The product is not soluble in water, thus, in this case, water behaves as an antisolvent. More purified water (S-204) is pumped by and cooled by (RV203 ) to $5-10{ }^{\circ} \mathrm{C}$, and added slowly to the reactor for 15 minutes. The reaction mixture is transferred by gravity into a receiving vessel (RV-101) and ethyl acetate (S-206) is pumped into the same receiving vessel where the mixture undergoes liquid-liquid extraction. The organic layer (S-207) is pumped back to the reactor while the aqueous layer (S-208) is sent for waste treatment. The residual solvents are removed from the reactor (R-102) under vacuum by the condenser, which is sent to solvent recovery in the same fashion as in Step 1.

\section{Process Simulation of Step 2}

The unit-operations in the sequence needed to simulate the Step 2 are shown in Table 2.

Table 2. Unit operations in Step 2

\begin{tabular}{|c|c|}
\hline $\begin{array}{c}\text { Equipment } \\
\text { Tag }\end{array}$ & Unit Operation \\
\hline \multirow{4}{*}{ R-101 } & Transfer in \\
\cline { 2 - 2 } & Batch Vaporization \\
\cline { 2 - 2 } & Charge (KOH) \\
\cline { 2 - 2 } & Charge (Butanol) \\
\cline { 2 - 2 } & Batch Heating \\
\cline { 2 - 2 } & Batch Stoich. Reaction \\
\cline { 2 - 2 } & Batch Cooling (Chilled Water) \\
\cline { 2 - 2 } & Batch Stoich. Reaction \\
\cline { 2 - 2 } & Transfer Out \\
\cline { 2 - 2 } & In Place Cleaning (CIP-1) \\
\hline \multirow{4}{*}{ RV-102 } & Transfer In \\
\cline { 2 - 2 } & Store \\
\hline RV-101 & Mixer - Settler Extraction \\
\hline
\end{tabular}

The excess solvent from the first step had to be transferred to the receiving vessel (RV-102) before the next reaction was initiated, for the purpose of optimizing the reactor volume. This was simulated by using Batch Vaporization operation. The components needed to be evaporated were specified, as well as the percentage of their removal. The reactor (R101) was shared between the procedures for Step 1 and Step 2, and this was specified in the reactor data, and also taken into account when scheduling the various operations. In order to schedule these reaction steps in AspenBatch software, the operations had to be specified as parallel tasks. The reaction stoichiometry was specified in a similar manner as in the Step 1, and the Intermediate II was produced (the conversion was 94\%). The In Place Cleaning operation was scheduled after the reactor content was transferred to the receiving vessel (RV-102). Methanol was chosen as a cleaning agent, and the duration of cleaning was also specified. As mentioned in the process description the reaction mixture is refluxed by using condenser for three hours, and this is a quite frequent way of achieving the desired conversion in pharmaceutical processes. However, this operation was not available in any of the software packages considered in this project. The extraction was simulated in the same way as in Step 1; the partition coefficients for several components were selected. For the purpose of the simplicity of the calculation, the total separation of the desired component was assumed, and the partition coefficients assigned accordingly.

\subsection{Resolution}

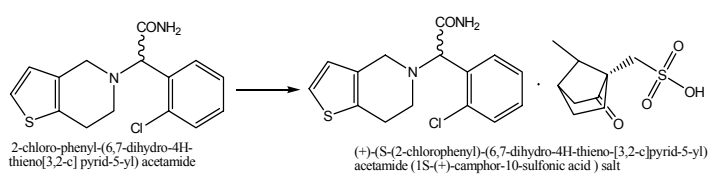

\section{Scheme 3. Reaction Mechanism for Resolution (Step 3)}

The excess of ethyl acetate that comes in the reactor with the Intermediate II is first removed by vacuum into the receiving vessel (RV-103). Acetone (S-301) and CSA-acetone solution (S302) are pumped, into the reactor (R-102) containing the product from the previous reaction. This addition is done at $15-20{ }^{\circ} \mathrm{C}$ over the course of 4 hours. After the reaction is completed, the solvents are removed from the reaction mixture under reduced pressure by condenser (C-101) and the reactor jacket is cooled to below $8{ }^{\circ} \mathrm{C}$ to encourage precipitation of the product. The slurry (S-303) is transferred 
via gravity to a filter (F-101) where it is filtered and washed twice with acetone (S-304). The filtrate (S-306) is sent to the waste treatment and the filter cake $(\mathrm{S}-305)$ is transferred to the second reactor (R-101).

\section{Process Simulation of Step 3}

The operations in the sequence needed to simulate the Step 3 are shown in Table 3.

Table 3. Unit operations for Step 3

\begin{tabular}{|c|c|}
\hline Equipment Tag & Unit Operation \\
\hline \multirow{10}{*}{$\mathrm{R}-102$} & Transfer in \\
\hline & Agitation \\
\hline & Batch Vaporization \\
\hline & Charge (CSA) \\
\hline & Charge (Acetone) \\
\hline & Batch Stoich. Reaction \\
\hline & Batch Vaporization \\
\hline & Batch Cooling ( $\mathrm{NaCl}$ Brine) \\
\hline & Transfer Out \\
\hline & In Place Cleaning (CIP-1) \\
\hline \multirow{3}{*}{ RV-103 } & $\begin{array}{c}\text { Transfer In (Ethyl Acetate }+ \\
\text { Residuals from Step 2) } \\
\end{array}$ \\
\hline & Transfer In (Acetone) \\
\hline & Store \\
\hline \multirow{5}{*}{ F-101 } & Transfer In \\
\hline & Cloth Filtration \\
\hline & Cake Wash (Acetone) \\
\hline & Transfer Out \\
\hline & In Place Cleaning (CIP-1) \\
\hline
\end{tabular}

The Batch Vaporization was used twice in this step to simulate the removal of solvents under reduced pressure. The reduced pressure of 50 $\mathrm{KPa}$ was specified, and the components to be removed were specified as well. The first time, the excess of the solvent from the extractor was vaporized, and the second time acetone was removed from the reaction mixture. The stoichiometric reaction was simulated using the conversion of $60 \%$ of the Intermediate II, which was a reference component. The product of Step 3 was filtered using Nutshce filter unit procedure. The cake thickness as well as the cake dryness had to be specified in order to simulate the procedure. The cake was also washed with acetone.

\subsection{Freebasing to the Amide}

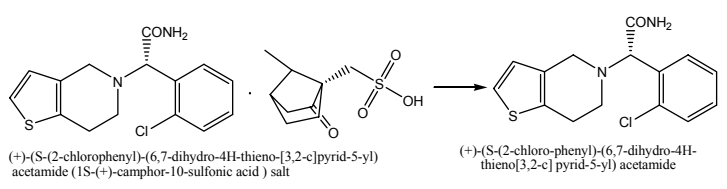

\section{Scheme 4. Reaction Mechanism for Freebasing to the Amide (Step 4)}

Once the solid product from Step 3 is transferred to the second reactor (R-102), purified water (S$403)$ and sodium carbonate solution (S-401) are added. This mixture is stirred for 2 hours and after the reaction is completed, ethyl acetate (S402 ) is pumped into the reactor for extraction of the product. The reaction occurs at $40{ }^{\circ} \mathrm{C}$. The aqueous layer contains the undesired (R) enantiomer and is removed from the bottom of the reactor (S-404) where it may undergo further purification. The solvents from the organic layer are removed by condenser $(\mathrm{C}-101)$ and sent to the receiving vessel $(\mathrm{RV}-104)$ via $(\mathrm{S}-405)$. This process results in obtaining the solid product (Senantiomer).

\section{Process Simulation for Step 4}

The operations in the sequence needed to simulate the Step 4 are shown in Table 4.

Table 4. Unit operations for Step 4

\begin{tabular}{|c|c|}
\hline Equipment Tag & Unit Operation \\
\hline \multirow{4}{*}{ R-102 } & Transfer in \\
\cline { 2 - 2 } & Agitation \\
\cline { 2 - 2 } & Charge (Sodium Carbonate) \\
\cline { 2 - 2 } & Charge (Water) \\
\cline { 2 - 2 } & Batch Stoich. Reaction \\
\cline { 2 - 2 } & Charge (Ethyl Acetate) \\
\cline { 2 - 2 } & Batch Extraction (Phase Split) \\
\cline { 2 - 2 } & Transfer Out \\
\hline \multirow{4}{*}{ RV-104 } & Transfer In \\
\cline { 2 - 2 } & Store \\
\hline
\end{tabular}

The reaction conversion was $60 \%$ based on Intermediate III as a reference component. The excess solvent is removed in this case using Batch Extraction / Phase Split for the separation of the Intermediate IV. The percentage of component removal was specified in the model 
and the undesired components were transferred into the receiving vessel (RV-104).

\subsection{Methylation}

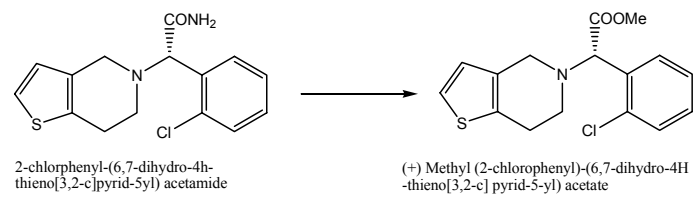

\section{Scheme 5. Reaction Mechanism for Methylation (Step 5)}

Methanol (S-501) is pumped by to the second reactor (R-101) containing the solid product from the previous step and stirred. Once the product is dissolved concentrated sulfuric acid (S-502) was added in portions via to the reaction mixture over a period of 1.5 hours. This reaction mixture is then refluxed at $80{ }^{\circ} \mathrm{C}$ for 26 hours using condenser to obtain desired conversion. Note, this information was taken from the patent, and those experiments have not been optimized. It is also applicable to the lab scale, but it is most likely different in the pilot plant. After the reaction is complete the residual solvents are removed under reduced pressure. A solution of $\mathrm{KOH}(\mathrm{S}-504)$ is pumped into the reactor and mixed for 0.5 hours. This solution is filtered ( $\mathrm{F}$ 101) and the filtrate is sent back to the reactor using a pump and the solid waste from the cake (S-506) is sent to waste treatment. The aqueous layer is separated in the reactor and sent to waste treatment via (S-508). The remaining solvents are removed under vacuum, and the oily product is obtained and sent back to (R-101) via (S-509).

\section{Process Simulation for Step 5}

The unit-operations in the sequence needed to simulate the Step 5 are shown in Table 5.

The charge operations as well as the scheduling of the various operations were performed in the similar manner as in the previous steps.

The reaction extent was $64 \%$ based on Intermediate IV as the reference component. The excess solvent was removed using Batch Vaporization operation under reduced pressure of $50 \mathrm{KPa}$.
Table 5. Unit operations in Step 5

\begin{tabular}{|c|c|}
\hline Equipment & Unit Operation \\
\hline \multirow{4}{*}{ R-101 } & Transfer in \\
\cline { 2 - 2 } & Agitation \\
\cline { 2 - 2 } & Charge (MeOH) \\
\cline { 2 - 3 } & Charge (Sulfuric Acid) \\
\cline { 2 - 2 } & Charge (KOH) \\
\cline { 2 - 2 } & Batch Stoich. Reaction \\
\cline { 2 - 3 } & Batch Vaporization \\
\cline { 2 - 2 } & Batch Cooling (Chilled Water) \\
\cline { 2 - 2 } & Transfer Out \\
\hline \multirow{5}{*}{ F-101 } & Tlace Cleaning (CIP-1) \\
\cline { 2 - 2 } & Charge (Acetone) \\
\cline { 2 - 2 } & Cloth Filtration \\
\cline { 2 - 2 } & In Place Cleaning (CIP-1) \\
\hline
\end{tabular}

The reactor (R-101) is shared between several steps, and this was also specified when scheduling the operations in various sections. The In Place Cleaning operation was scheduled after the reactor content was transferred to the filter (F-101). Methanol was chosen as a cleaning agent, and the duration of cleaning was also specified.

\subsection{Acid Addition}

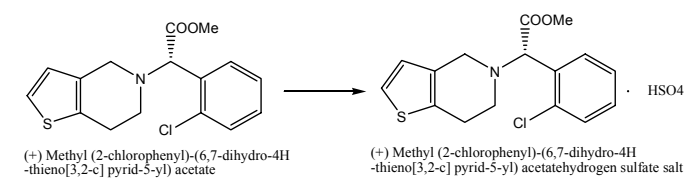

\section{Scheme 6. Reaction Mechanism for Acid Addition (Step 6)}

Ice-cold acetone (S-601) is pumped and cooled by (RV-602) into the reactor (R-101). Acetone is used to dissolve clopidogrel freebase made in the last step. Concentrated sulfuric acid (S-602) is also pumped and cooled by (P-603) and (RV603). The reaction occurs at $40{ }^{\circ} \mathrm{C}$. After desired conversion is obtained, the reaction mixture is cooled down to $5{ }^{\circ} \mathrm{C}$ to enhance crystallization of the CPG bisulfate. Water by itself is not a sufficient coolant to obtain this temperature inside the reactor, and thus the mixture of methanol and water used as a coolant for this reaction step. The reaction mixture $(\mathrm{S}-605)$ is sent to a filter $(\mathrm{F}-101)$ to separate the solid product and the filter cake is washed twice with 
acetone (S-604). The solid is sent to a vacuum rotary cone dryer $(\mathrm{D}-101)$ at $25^{\circ} \mathrm{C}$ where it is dried by contact with the hot surface of the dryer walls. The acetone vapour is removed by the vacuum pump and condensed.

\section{Process Simulation for Step 6}

The unit-operations in the sequence needed to simulate the Step 6 are shown in Table 6 .

The reactive crystallization occurs in Step 6, and this was still simulated by using Batch Stoichiometric Reaction operation and Crystallization operation in series. This was done in order to obtain the product first with the conversion of $100 \%$, and then to indicate the formation of solids. The crystal form component was indicated, and the final temperature of $5^{\circ} \mathrm{C}$. The cleaning of the reactor was simulated using In Place Cleaning operation, so that the reactor is ready for the next running the next batch.
Table 6. Unit operations for Step 6

\begin{tabular}{|c|c|}
\hline Equipment Tag & Unit Operation \\
\hline \multirow{10}{*}{$\mathrm{R}-102$} & Transfer in \\
\hline & Charge (Acetone) \\
\hline & Agitation \\
\hline & Batch Heating \\
\hline & Charge (Sulfuric Acid) \\
\hline & Batch Stoich. Reaction \\
\hline & Crystallization \\
\hline & Batch Cooling \\
\hline & Transfer Out \\
\hline & In Place Cleaning (CIP-1) \\
\hline \multirow{5}{*}{ F-101 } & Transfer In \\
\hline & Cloth Filtration \\
\hline & Cake Wash (Acetone) \\
\hline & Transfer Out \\
\hline & In Place Cleaning (CIP-1) \\
\hline \multirow{3}{*}{ D-101 } & Transfer In \\
\hline & Charge \\
\hline & Drying \\
\hline
\end{tabular}

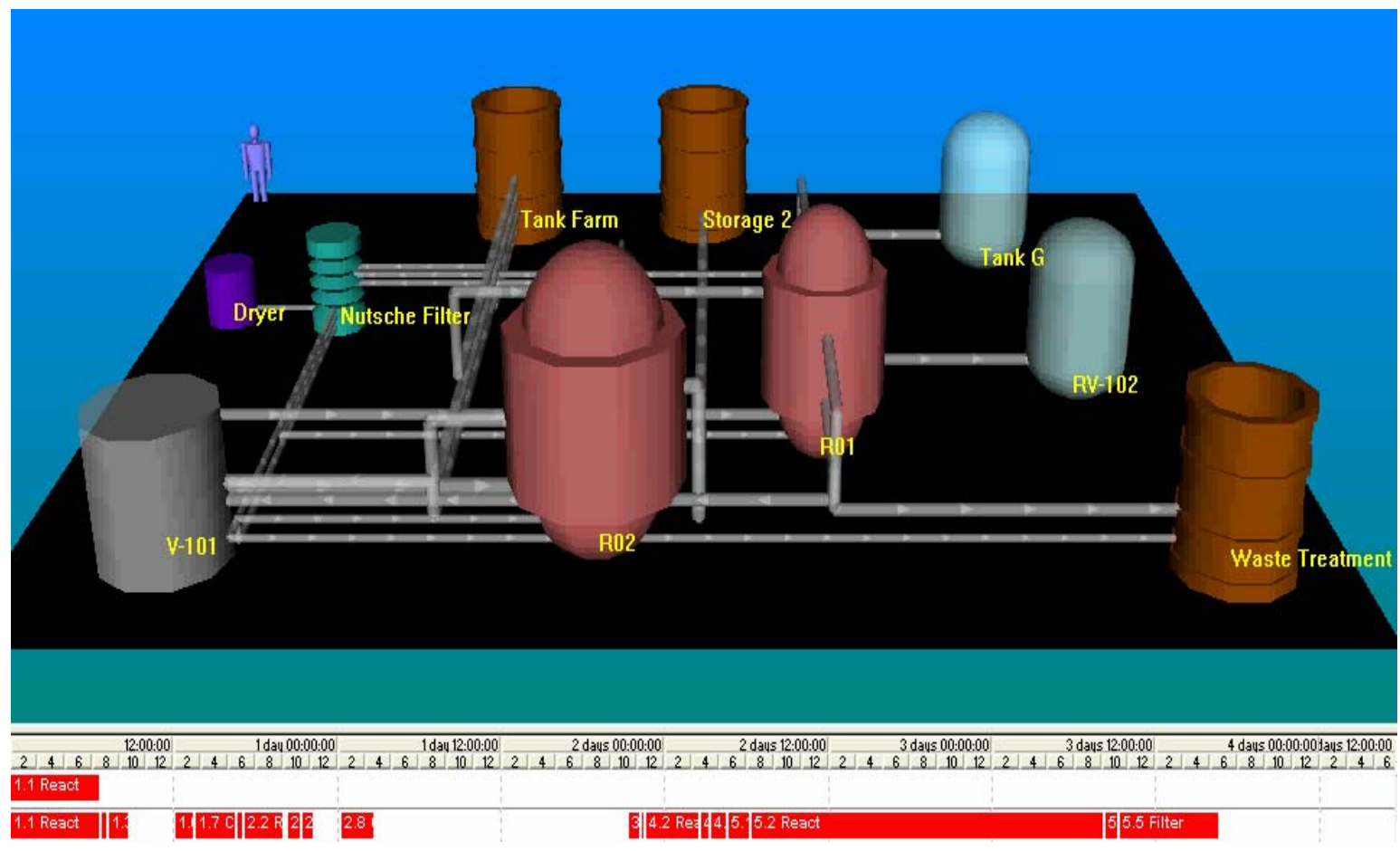

Figure 3. 3D picture of plant for manufacturing of CPG from AspenBatch(TM) 
The excess of the solvent was vaporized under the pressure of $50 \mathrm{KPa}$. The final product of was filtered using Nutshce filter unit procedure. The cake thickness as well as the cake dryness had to be specified in order to simulate this procedure. The cake was also washed with acetone. Finally, the product was dried using Double Cone Dryer unit procedure. In the real process the drying is performed under vacuum, but this unit procedure was not available in any of the two software packages.

As mentioned above, AspenBatch ${ }^{\mathrm{TM}}$ is a recipe driven software package. However, one of the unique output options is the $3 \mathrm{D}$ simulation of the process after the model is completed. Figure 3 depicts three-dimensional picture of the described process.

\section{Simulation Results}

\subsection{Material and Stream Balances}

The software packages track material balances across the entire process. The solution of material and energy balances involves the calculation of the flowrate, composition and thermodynamic state of all streams and equipment contents in the flowsheet.

The advantage of SPD over other batch software packages used for this project was that material balance results are easily accessed by selecting the stream of interest in the processflow diagram. This opens a window with the detail description of the composition, flowrate and density of the stream. However, AspenBatch ${ }^{\mathrm{TM}}$ has a powerful tool for predicting physical and thermodynamic properties based on the compound's chemical structure.

The generated report for material and stream balances contains the overall material balance, the stream material balance, the material balance related to separate sections, raw materials, and the equipment contents.

The overall process data and the raw material requirements are shown below in Tables 7

\section{Table 7. Overall process data (from SPD software package)}

\begin{tabular}{|lc|}
\hline Annual Operating Time & 249.70 day \\
Annual Throughput & $8,784.00 \mathrm{~kg} \mathrm{MP}$ \\
Batch Throughput & $244.00 \mathrm{~kg} \mathrm{MP}$ \\
Recipe Batch Time & 5.65 day \\
Recipe Cycle Time & 6.97 day \\
Number of Batches per Year & 36.00 \\
MP = Main Product = Total Flow in S-609 (CPG BS)
\end{tabular}

\subsection{Scheduling}

Both software packages perform process scheduling and generate the Gantt chart, which is shown in Figure 4.

\begin{tabular}{|c|c|c|c|c|c|c|c|c|c|c|c|}
\hline & Duration & 1 & 2 & 3 & & 4 & 5 & & 6 & 7 & 8 \\
\hline Task & (h) & \begin{tabular}{l|l}
16 & \\
\end{tabular} & 48 & 64 & 80 & 96 & \begin{tabular}{|l|l|}
112 &
\end{tabular} & 128 & 144 & \begin{tabular}{l|l}
160 & \\
\end{tabular} & 176 \\
\hline$\boxminus$ Complete Recipe & 146.23 & & & & & & & & & $(0.00 \mathrm{~h}-1)$ & $146.23 \mathrm{~h}, 146.23 \mathrm{~h})$ \\
\hline ÐP-1 in R-101 & 15.30 & & & & & & & & & & \\
\hline 円P-2 in RV-101 & 3.17 & & & & & & & & & & \\
\hline ÐP-3 in R-101 & 17.92 & & & & & & & & & & \\
\hline 田-4 in RV-102 & 6.35 & & & & & & & & & & \\
\hline 円P. 5 in RV-101 & 1.42 & & & & & & & & & & \\
\hline 田-6 in R-102 & 23.58 & & & & & & & & & & \\
\hline ĐP.7 in RV-103 & 11.99 & & & & & & & & & & \\
\hline Ð P-8 in F-101 & 4.33 & & & & & & & & & & \\
\hline ÐP-9 in R-101 & 12.60 & & & & & & & & & & \\
\hline ÐP-10 in RV-104 & 6.84 & & & & & & & & & & \\
\hline ÐP-11 in R-101 & 56.53 & & & & & & & & & & \\
\hline 田-12 in F-101 & 7.17 & & & & & & & & & & \\
\hline ÐP -13 in R-102 & 11.80 & & & & & & & & & & \\
\hline ÐP-14 in F-101 & 2.42 & & & & & & & & & & \\
\hline ÐP-15 in DCDR-101 & 6.75 & & & & & & & & & & \\
\hline
\end{tabular}

Figure 4. Gantt chart for manufacturing of CPG 
Note that in this Gantt chart, only the main procedures were shown, but there is an option of showing each operation within the specific procedure. As seen from the Figure 6, the longest procedure is $\mathrm{P}-11$ (Methylation Reaction) in the reactor (R-101). This indicates that the main bottleneck of the entire process is in this procedure, and that main optimization of the process should be addressed to reduce the duration of this procedure. It can be also seen that there is an overlap between some of the steps. This is due to the cleaning in place operation of one reactor, while the reaction for the following step is carried out in the other reactor.

\section{Process Economics}

Economic analysis was performed using the SPD software package. The purchase cost is first factor for cost estimation. This can be evaluated either by using built-in model in SPD which is working based on Power law correlation or entering known value for all equipments. The total capital cost is sum of the direct cost (DC), indirect cost (IC) and other cost (OC).

Some of the main factors for annual operating calculation are as follows:

1. Labor Costs: It is sum of the itemized estimate for operating labor on a stepby-step process and additional labor defined on lumped time basis.

2. Laboratory/QC (Quality Control)/ QA (Quality Assurance): It is equal to 15\% of total labor cost.

3. Raw materials

4. Utilities: It includes all the costs for electricity and heat transfer agents (steam, cold/chilled water and $\mathrm{NaCl}$ brine).

5. Facility Dependant Cost: Main factors for this item are maintenance, depreciation, insurance, local taxes and factory expenses.

6. Waste Treatment and disposal Cost: CPG-BS plant has organic and aqueous wastes, solid disposals and emission and annual operating cost for processing these material should be considered in this item.

The final step in the economic analysis was to calculate the profitability of the proposed plant. This was done using SuperPro Designer ${ }^{\mathrm{TM}}$ economic estimator and simply took the total revenues generated by the product and subtracted the costs associated with manufacturing the product. The Research and Development (R\&D) represents an important cost in any pharmaceutical industry and this cost was fixed for all process steps. The gross margin of the project was calculated $72.59 \%$, and the payback period for this plant was found to be a little over 1.38 years.

SPD generated detail reports for total capital investment, annual operating cost and profitability which are very helpful for tracking results and detail analysis. Table 8 shows the executive summery of cost analysis for manufacturing CPG-BS project.

Table 8. Executive summery of economic evaluation

\begin{tabular}{|c|c|}
\hline Total Capital Investment & $82,316,000 \mathrm{~S}$ \\
\hline Capital Investment Charged to This Project & $82,316,000 \mathrm{~S}$ \\
\hline Operating Cost & $61,158,000 \$$ /yr \\
\hline Production Rate & $8,763,271.58 \mathrm{~g} \mathrm{MP} / \mathrm{yr}$ \\
\hline Unit Production Cost & $6.98 \$ / g \mathrm{MP}$ \\
\hline Total Revenues & $156,441,000 \$ / y r$ \\
\hline Gross Margin & $60.91 \%$ \\
\hline Return On Investment & $72.59 \%$ \\
\hline Payback Time & 1.38 years \\
\hline IRR (After Taxes) & $46.95 \%$ \\
\hline NPV (at $7.0 \%$ Interest) & $356,787,000 \$$ \\
\hline $\mathrm{MP}=$ Total Flow of Stream CPG-BS & \\
\hline
\end{tabular}

The mathematical modeling of crystallization and drying were performed to improve the design.

Linear, polynomial, and exponential cooling profiles, as well as seed characteristics and initial conditions were studied to achieve the most favorable conditions for crystallization.

In order to avoid thermal degradation of the product, contamination of the product by hot gases, and loss of this expensive product, the vacuum rotary cone dryer was found to be the best selection. Due to the lack of the experimental data, the theoretical modeling (using Matlab ${ }^{\mathrm{TM}}$ ) of drying curves was performed in order to find the optimal design parameters. These parameters include vacuum pressure, wall temperature profile, and the speed of rotation of the dryer.

\subsection{Crystallization Model}

The crystallization model was based on solving the population balance and the mass 
balance by using Matlab ${ }^{\mathrm{TM}}$ programming [7]. The population balance equation for a batch crystallizer can be described by the following equation:

$$
\frac{\partial f(L, t)}{\partial t}+\frac{\partial(G(L, t) f(L, t))}{\partial L}=0
$$

A mass balance for batch crystallization can be expressed as:

$$
\frac{d C}{d t}=-\rho k_{v} \frac{d \mu_{3}}{d t}
$$

The solubility of CPG in the solvent (Acetone) was determined experimentally, and this data was considered in the model as well. The effect of temperature cooling profile on the crystal size distribution was investigated. In this case, the reactive crystallization is used to convert $\mathrm{CPG}$ freebase to CPG bisulfate, which is a more stable form. The reaction occurs between CPG freebase and sulfuric acid, which results in production of fine CPG particles and saturated solution. For the purpose of modeling, it was assumed that these fine solid particles in the solution were acting as a seed for the crystal growth. It was also assumed that the size distribution of these fine crystals is between 5 to $50 \mu \mathrm{m}$. With constant crystallization time and same seed size distribution for both cases, linear cooling profile gives larger particle size $(250-350 \mu \mathrm{m})$ than nonlinear profile $(150-200 \mu \mathrm{m})$. Based on desired crystal size distribution, temperature cooling profile can be selected.

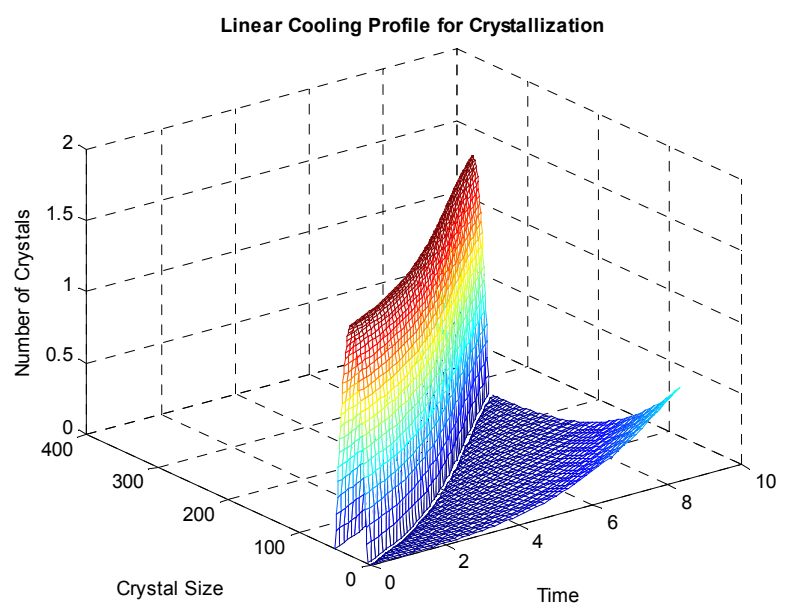

Figure 5. Linear Cooling Profile for Crystallization of CPG
Figure 5 shows the crystal size distribution of $\mathrm{CPG}$ in acetone for linear cooling profile.

It is important to mention that the final crystal size has direct effect on the filtering time and the cost.

\subsection{Dryer Model}

For pharmaceuticals, the use of vacuum contact drying is advantageous since this process allows for drying at a lower temperature for heat sensitive materials and results in virtually no loss of the expensive product. The generation of a standard drying rate curve is challenging with this type of dryer since typical equations and psychometric charts do not apply. Schlunder et al. [9] proposed a model based on Schlunder's 'penetration theory'. In this model a steady mixing process is replaced by a sequence of individual, intermittent mixing steps. The fact that mixing is not continuous improves the drying process in the sense that agglomeration is minimized and the drying front is regularly renewed. For the calculations, the product is assumed to be homogenously mixed, and the particle size reduction is neglected. The main assumptions of the model are as follows:

- $\quad$ the particles are spherical

- drying time includes two main states: dynamic and static

- the particles are dried until 0.001\% moisture

The complex set of heat and mass transfer calculations was solved using MATLAB program, which enabled using the iterative calculations and numerical integration when needed. After the program was compiled successfully, the software with the graphical user interface was created to make the program user friendly and practical for users that are not familiar with MATLAB programming. The software allows changing the properties of the material, as well as the operating conditions, which makes it useful for optimization and quick estimation of time needed for drying process and design parameters of the dryer. The software generates the output consisting of area, saturation temperature of solvent that is being removed, diameter of the cylinder section and height of the dryer. The software also provides a drying curve as well as the penetration depth and the normalized temperature difference as a function of time. The sample drying curve is shown in Figure 6. 


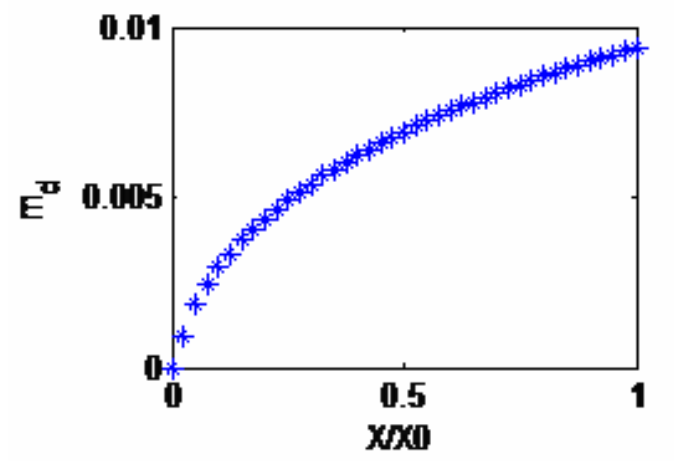

Figure 6. Drying curve as a function of moisture content

As seen in Figure 6, there is no constant drying rate period as it would be expected from conventional dryers. This can be explained by the fact that this is an unsteady process, which undergoes the intermittent rotation of the dryer.

\section{Conclusion}

Extensive work has been done to decipher a process from an existing patent and design a flexible plant not only for the manufacturing of Clopidogrel Bisulfate but also for other pharmaceutical products. The design of the reactor unit was performed in a way that it could accommodate five reaction steps as well as the crystallization process. The entire process has been successfully simulated using two different software packages, AspenBatch ${ }^{\mathrm{TM}}$ and SuperPro Designer ${ }^{\mathrm{TM}}$, and have both yielded similar results to those done manually. The crystallization model yielded that the liner cooling profile was the best approach for achieving the desired CSD. The drying model and the graphical user interface created by authors allowed an easy optimization of the drying process and the calculation of the dryer surface area. The project management and economic analysis performed by the two different software packages resulted in period of 1.38 years for the return on the investment.

\section{Acknowledgments}

We would also like to thank the Chemical Engineering Department for purchasing the SuperPro Designer ${ }^{\circledR}$ (Academic Site Edition) v. 6.0. for this project after the author's request. The help provided by Dr. David Stradiotto of Apotex Pharmachem has also been invaluable and the project engineers would also like to express their thanks to him as well.

\section{Nomenclature}

$\begin{array}{ll}\mathrm{C} & \text { Concentration } \\ \mathrm{G} & \text { Crystal growth rate } \\ \mathrm{L} & \text { Crystal size } \\ \mathrm{k}_{\mathrm{v}} & \text { Volume shape factor } \\ \mathrm{t} & \text { Time } \\ \rho & \text { Density } \\ \mu & \text { Moment of CSD }\end{array}$

\section{References}

[1] Silve, R.A., "Preparation of (S)- Clopidogrel and related compounds", 2003, (US patent: 6858734)

[2] Koradia, V., Chawla, G., \& Bansal, A.K. "Qualitative and quantitative analysis of clopidogrel bisulphate polymorphs". Acta Pharm, 54 (1), 2004, 193-204

[3] Evers, S.M., Struijs, J,N. \& Ament, A,J. "International comparison of stroke cost studies" Stroke 35 (6), 2004, 1209-1215.

[4] Robless, P., Mikhailidis, D. P.\& Stansby, G. "Systematic review of antiplatelet therapy for the prevention of myocardial infarction, stroke or vascular death in patients with

peripheral vascular disease". British Journal of Surgery 88 (6), 2001, 787-798

[5] Harker, Boissel, Pilgrim, Gent. "A randomized, blinded, trial of clopidogrel versus aspirin in patients at risk of ischemic events (CAPRIE)." The Lancet 348 (9038), 1996, 1329-1339.

[6] Gerschutz, G.P. \& Deepak, B.L.. "The CURE trial: Using clopidogrel in acute coronary syndromes without ST-segment elevation." Cleveland Clinic Journal of Medicine 69 (5), 2003,377-385.

[7] Hu, Q., Rohani, S. \& Jutan, A.. "Modeling and optimizing of seeded batch crystallizers". Comp. and Chem Eng 68, (5), 2004125-135.

[8] Petrides D. P., Koulouris A., Lagonikos P. T. “ The Role of Process Simulation in Pharmaceutical Process Development and Product Commercialization" Pharmaceutical Engineering 22 (1), 2002

[9] Schlunder, E.U., \& Mollekopf, N. "Vacuum Contact Drying of Free Flowing Mechanically Agitated Particulate Material". Chem. Eng. Process 78, (51), 1983, 1345-1360. 